\title{
Real-World Data for Lenvatinib in Radioiodine-Refractory Differentiated Thyroid Cancer (RELEVANT): A Retrospective Multicentric Analysis of Clinical Practice in Austria
}

\author{
G. Rendl $\left(\mathbb{D},{ }^{1}\right.$ B. Sipos $\left(\mathbb{D},{ }^{1}\right.$ A. Becherer $(D),{ }^{2}$ S. Sorko, ${ }^{3}$ C. Trummer $\left(\mathbb{D},{ }^{4}\right.$ M. Raderer $(D),{ }^{5}$

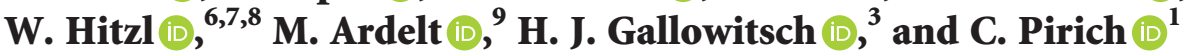 \\ ${ }^{1}$ Department of Nuclear Medicine and Endocrinology, University Hospital Salzburg, Paracelsus Medical University Salzburg, \\ Salzburg, Austria \\ ${ }^{2}$ Department of Nuclear Medicine, Academic Teaching Hospital Feldkirch, Feldkirch, Austria \\ ${ }^{3}$ Department of Nuclear Medicine and Endocrinology, PET/CT Centre, Klinikum Klagenfurt am Wörthersee, Klagenfurt, Austria \\ ${ }^{4}$ Division of Endocrinology and Diabetology, Department of Internal Medicine, Medical University of Graz, Graz, Austria \\ ${ }^{5}$ Department of Internal Medicine I, Division of Oncology, Medical University of Vienna, Vienna, Austria \\ ${ }^{6}$ Research Office (Biostatistics), Paracelsus Medical University Salzburg, Salzburg, Austria \\ ${ }^{7}$ Department of Ophthalmology and Optometry, University Hospital Salzburg, Paracelsus Medical University Salzburg, \\ Salzburg, Austria \\ ${ }^{8}$ Research Program Experimental Ophthalmology and Glaucoma Research, University Hospital Salzburg, \\ Paracelsus Medical University Salzburg, Salzburg, Austria \\ ${ }^{9}$ Institute of Pharmacy, Paracelsus Medical University Salzburg, Salzburg, Austria
}

Correspondence should be addressed to G. Rendl; g.rendl@salk.at

Received 30 July 2020; Revised 3 November 2020; Accepted 9 November 2020; Published 29 November 2020

Academic Editor: Giorgio Borretta

Copyright $\odot 2020$ G. Rendl et al. This is an open access article distributed under the Creative Commons Attribution License, which permits unrestricted use, distribution, and reproduction in any medium, provided the original work is properly cited.

\begin{abstract}
Background. Lenvatinib has proven efficacy in progressive, radioiodine- (RAI-) refractory thyroid cancer (TC). Dose reductions are commonly performed due to decreased tolerability and adverse effects. This retrospective multicenter study analyzed overall survival (OS) and progression-free survival (PFS) and tolerability in the Austrian patient population treated with lenvatinib. Methods. Clinical data of 43 patients (25 males and 18 females) with a median age of 70 years (range: 39-91 years) and RAIrefractory TC with metastases to the lymph nodes (74\%), lungs ( $86 \%)$, bone (35\%), liver (16\%), and brain (12\%) were analyzed. The mean duration of treatment with lenvatinib was $26.6 \pm 15.4$ months with dosage reductions required in 39 patients (91\%). Results. PFS after 24 months was 71\% (95\% CI: 56-87), and overall survival (OS) was 74\% (95\% CI: 60-88), respectively. OS was significantly shorter $(p=0.048)$ in patients with a daily maintenance dosage $\leq 10 \mathrm{mg}(63 \%)(95 \% \mathrm{CI}$ : $39-86)$ as compared to patients on $\geq 14 \mathrm{mg}$ lenvatinib (82\%) (95\% CI: 66-98) daily. Dose reduction was noted in 39 patients (91\%). Grade $\geq 3$ toxicities (hypertension, diarrhea, weight loss, and palmar-plantar erythrodysesthesia syndrome) were most common leading to discontinuation of lenvatinib in 7 patients (16\%). Conclusion. Lenvatinib showed sustained clinical efficacy in patients with metastatic RAI-refractory TC even with reduced maintenance dosages over years. The effects were comparable to the registration trial, although patients had a higher median age and, more commonly, dose reductions.
\end{abstract}

\section{Introduction}

Thyroid cancer (TC) is the most common endocrine malignancy, with a rising incidence worldwide. According to Statistik Austria, 822 cases of TC (9.2 per 100,000 people) were diagnosed in 2016 of whom 78 patients died (0.9 per 100,000 people) $[1,2]$. Histopathologically, three types of TC exist: differentiated thyroid cancer (DTC), medullary thyroid cancer, and anaplastic thyroid cancer [3]. DTC represents the vast majority of thyroid cancer cases (>90\%), 
including both papillary and follicular forms [3, 4]. In 2013, the percentage of the papillary form amounted to $84 \%$ among newly diagnosed TC within the federal state of Salzburg, Austria [5].

In terms of standardized treatment, surgical removal of the thyroid gland, i.e., thyroidectomy, followed by radioiodine ablation (RAI) and thyroid-stimulating hormone suppression therapy with levothyroxine has been the standard of care for all DTC, except for pTla in Austria [6]. According to the guideline of the European Association of Nuclear Medicine, an ablation of any residual thyroid remnant tissue following thyroidectomy with RAI enables the follow-up of the patient by the use of the biomarker thyroglobulin and decreases the incidence of recurrent disease [7].

The majority of patients with DTC responds well to this multimodal standard treatment regimen; however, up to $15 \%$ of DTC cases develop RAI-refractory disease [8-10]. RAI-refractory disease is associated with an increased risk of death from DTC $[8,9]$, though its definition is not uniform among national and international guidelines and recommendations published $[6,9,11,12]$.

In a landmark retrospective study, patients with RAIrefractory disease exhibit a 10 -year survival rate of only $10 \%$ from the time of detection of metastases [8, 9], therefore often requiring a multimodular treatment regimen which includes systemic therapies [11].

The therapeutic strategy for the treatment of radioiodinerefractory thyroid cancer has been changing with market approval and introduction of tyrosine kinase inhibitors (TKIs). The DECISION trial employing Sorafenib was first used to demonstrate a significant prolongation in progression-free survival (PFS) in radioiodine-refractory DTC [13].

Lenvatinib is an oral multitargeted TKI of vascular endothelial growth factor receptor (VEGFR) 1-3, fibroblast growth factor receptor (FGFR) 1-4, platelet-derived growth factor receptor- (PDGFR-) $\alpha$, and RET and KIT proto-oncogenes $[14,15]$. Lenvatinib was approved for the treatment of radioiodine-refractory DTC in the United States and Europe $[16,17]$ based on the results of the pivotal phase-3 SELECT study [18].

However, the benefit of systemic treatment with TKI must outweigh the potential harm of this class of drugs since a substantial proportion of patients might present without symptoms and with minor degree of disease progression for years [19].

Therefore, national guidelines have been introduced to define the patient population which might benefit most from the initiation of systemic treatment with the TKI $[6,12]$.

The aim of our retrospective multicenter study was to analyze (1) the Austrian patient population treated with lenvatinib after its European approval, (2) the efficacy, and (3) the tolerability and toxicity of this treatment with regard to the profile and severity of adverse effects and their impact on maintenance dose.

\section{Materials and Patients}

This study included 43 patients (18 female and 25 male patients) with a mean age of 67 years \pm 11 years (median age: 70 years; range: 39-91 years) from five nuclear medicine, endocrine, or oncologic departments in Austria located in Salzburg $(n=13)$, Feldkirch $(n=7)$, Klagenfurt $(n=11)$, Graz $(n=3)$, and Vienna $(n=9)$.

This study was approved by the leading ethics committee of the Federal Republic of Salzburg and, subsequently, if required, by the local ethics committee.

The age at initial diagnosis of thyroid carcinoma was $60 \pm 13$ years (median age: 63 years; range: 33-90 years).

The majority of patients had DTC (84\%) (Table 1). Initial treatment consisted of thyroidectomy in 42 out of 43 patients, and 41 patients (95\%) had at least 1 RAI therapy.

Eleven patients underwent postoperative local radiotherapy of the thyroid bed (26\%), with a mean dose of 57 Gray (Gy) \pm 7 Gy (median dose: $60 \mathrm{~Gy}$; range: 45-66 Gy), and 12 patients (28\%) had radiotherapy of metastases. Nine out of 12 patients had external beam radiation therapy of metastatic bone lesions, 4 patients of metastatic pulmonary lesions, 2 patients had palliative radiotherapy of lymph node metastases, and one patient of hepatic metastasis, respectively. No patient underwent local radiotherapy during lenvatinib treatment.

In all patients, treatment initiation with a TKI such as lenvatinib was based on progressive disease in imaging (RECIST 1.1 [20]; PERCIST [21]) and by subsequent approval by an interdisciplinary tumor board consisting of specialists in radiation oncology, nuclear medicine, surgery, pathology, and oncology [6].

Eight patients (19\%) had prior TKI treatment, of whom six were on Sorafenib and two on pazopanib, respectively (Table 1). Progression of disease, mostly lung metastasis (86\%), in imaging was the only cause for switching from conventional therapy or $1^{\text {st }}$-line TKI therapy to lenvatinib.

Mean duration of disease until initiation of lenvatinib with a starting dose of $24 \mathrm{mg}$ daily was $7.1 \pm 6.0$ years (median: 5.9 years; range: $0.1-23.2$ years), and mean patient age at start of lenvatinib was $67 \pm 11$ years (median: 70 years; range: 39-91 years).

2.1. Standard Clinical Care. Symptoms, blood pressure and vital signs, and laboratory testing including levels of thyroglobulin (TG), thyroid-stimulating hormone (TSH), and thyroglobulin antibodies ( $\operatorname{Tg} \mathrm{Ab})$, as well as urinary analysis, were routinely recorded during follow-up, respectively.

After initiation of lenvatinib, follow-up visits were scheduled at 1-month intervals. Adverse effects were assessed according to the National Cancer Institute Common Terminology Criteria for Adverse Events (version 5) [22]. Dose reductions were performed due to treatmentrelated adverse effects at regularly scheduled follow-up visits. 3-month intervals were applied for patient monitoring in case of stable course of disease and their well-being.

Imaging was performed at 3- to 6-month intervals or in case of suspected progression of disease. Imaging consisted of computed tomography (CT) imaging in Vienna and Graz and F-18 fluorodeoxyglucose positron emission tomography/computed tomography (FDG PET/CT) imaging in all other centers.

In all centers, TG levels were evaluated $1,3,12$, and 24 months after beginning of lenvatinib therapy, respectively. 
TABle 1: Patient characteristics.

\begin{tabular}{lc}
\hline & $N=43$ \\
\hline Median age (years, range) & $70(39-91)$ \\
Female $(n(\%))$ & $18(42)$ \\
Histologic subtype $(n(\%))$ & \\
Papillary & $10(23)$ \\
Follicular & $26(61)$ \\
Poorly differentiated & $4(9)$ \\
Anaplastic and follicular & $2(5)$ \\
Anaplastic & $1(2)$ \\
Metastatic lesions $(n(\%))$ & \\
Pulmonary metastases & $37(86)$ \\
Bone metastases & $15(35)$ \\
Lymph node metastases & $32(74)$ \\
Liver metastases & $7(16)$ \\
Brain metastases & $5(12)$ \\
Soft tissue metastases & $11(26)$ \\
Prior radioiodine therapy $(n(\%))$ & \\
None & $2(5)$ \\
One & $10(23)$ \\
Two & $13(30)$ \\
Three & $7(16)$ \\
Four & $6(14)$ \\
More than four & $5(12)$ \\
Median radioiodine activity $(\mathrm{MBq}$, range) & $11100(1780-41277)$ \\
Prior TKI therapy & \\
Yes & $8(19)$ \\
No & $35(81)$ \\
\hline
\end{tabular}

Response to therapy was defined as the absence of tumor progression in imaging (RECIST 1.1 or PERCIST) and absence of clinical complications due to tumor progression.

2.2. Laboratory Methods. Standard laboratory methods were employed in all centers. All patients underwent standardized clinical workup with assessment of clinical status, body weight, blood pressure, and measurement of free thyroid hormones (free $\mathrm{T} 3$ and free $\mathrm{T} 4$ ), $\mathrm{TG}$, and $\mathrm{TgAb}$, electrolytes, albumin, liver and renal function, red and white blood cell, and platelet count. Standard urinary analysis was performed regularly, mainly for the assessment and course of micro- or macroproteinuria.

2.3. Data Collection and Statistical Analysis. Source data collection was performed at each center by the use of individual (electronic) patient records employing a standardized Microsoft Office Excel data sheet (Microsoft Corporation, Redmond, WH, USA). Data were anonymized and transferred for analysis to the statistical department of the Research Office of the Paracelsus Medical University Salzburg, Austria. Data were checked for consistency and normality. Fisher's exact test and Pearson's test were used to analyze cross-tabulations and distributions (age, sex, histology, and radioiodine therapies). One-factorial ANOVA and corresponding LSD test were used for pairwise comparisons. Kaplan-Meier analysis and Cox regression models were used for survival analysis. 95\% confidence intervals were computed for survival analyses and thyroglobulin levels under therapy. All reported tests were two-sided, and $p$ values $<0.05$ were considered statistically significant. All statistical analyses in this report were performed by the use of NCSS (NCSS 10, NCSS, LLC, Kaysville, UT) and Statistica 13 (Hill, T. and Lewicki, P. Statistics: Methods and Applications. StatSoft, Tulsa, OK).

\section{Results}

3.1. Survival. At the time of data cutoff (September 30, 2019), the mean duration of lenvatinib therapy was $26.6 \pm 15.4$ months (median: 27.6 months; range: 1.2-60.0 months) at a mean observation period of $32.6 \pm 13.7$ months (median: 34.6 months; range: 4.1-60.0 months) in all patients. Ten patients (one of them with prior TKI therapy) had died after a mean duration of $9.5 \pm 7.5$ months (median: 5.9 months; range: 1.5-21.3 months) of lenvatinib, whereas seven patients discontinued lenvatinib treatment after a mean duration of $21.1 \pm 14.4$ months (median: 21.6 months; range: $1.2-37.2$ months). The remaining 26 patients were still on lenvatinib treatment at the time of data cutoff with a mean duration of $32.6 \pm 13.7$ months (median: 34.6 months; range: 4.1-60.0 months).

Overall survival (OS) after 24 months was 74\% (95\% CI: 60-88). The progression-free survival (PFS) after 24 months was $71 \%$ (95\% CI: 56-87). Median OS and PFS has not been reached, yet.

The OS was not influenced by tumor subtype (FTC, PTC, or poorly differentiated/anaplastic TC) in our patient collective $(p=0.66)$.

Five patients had brain metastases, of whom three died, with a median OS of 12 months (range: 1-22 months), and seven patients had liver metastases, of whom three also died, with a median OS of 32 months (range: 5-48 months). The median OS of the remaining 31 patients (4 of whom died) amounted to 26 months (range: $4-49$ months).

PERCIST showed partial remission (PR) in 9\% $(n=4)$, stable disease (SD) in $16 \%(n=7)$, and progressive disease (PD) in $28 \%(n=12)$ of the 43 patients. One patient died after 3 months without restaging FDG PET/CT after initiation of lenvatinib therapy. No data were given for the remaining 19 patients (44\%) -7 of these patients were evaluated with PERCIST and 12 patients with RECIST 1.1.

3.2. Adverse Events. The incidence of treatment-related adverse effects of grade 3 or higher was $44 \%$. Higher-grade adverse events that were recorded in our patient collective were hypertension (any grade, 71\%; grade $\geq 3,26 \%$ ), weight loss (any grade, $70 \%$; grade $\geq 3,7 \%$ ), diarrhea (any grade, 44\%; grade $\geq 3,9 \%$ ), and palmar-plantar erythrodysesthesia syndrome (any grade, $14 \%$, grade $\geq 3,2 \%$ ) (Table 2). In case of grade $\geq 3$ adverse events, immediate dose reductions by at least $4 \mathrm{mg}$ lenvatinib daily were accomplished. No treatment-related deaths occurred in our patient collective. Persistent adverse events of any grade led to treatment discontinuation in $16 \%$ ( $n=7 \mathrm{pa}-$ tients). Drug holiday due to adverse events lasted no longer than 3 days in any case. 
TABLE 2: Overview of adverse events.

\begin{tabular}{|c|c|c|c|c|c|}
\hline Adverse events & All grades (\%) & Grade $1(\%)$ & Grade $2(\%)$ & Grade $3(\%)$ & Grade $4(\%)$ \\
\hline Hypertension & 71 & 19 & 26 & 26 & 0 \\
\hline Decreased weight & 70 & 30 & 33 & 7 & 0 \\
\hline Fatigue & 59 & 33 & 26 & 0 & 0 \\
\hline Proteinuria & 48 & 29 & 19 & 0 & 0 \\
\hline Diarrhea & 44 & 26 & 9 & 7 & 2 \\
\hline Decreased appetite & 23 & 14 & 9 & 0 & 0 \\
\hline Asthenia & 21 & 19 & 2 & 0 & 0 \\
\hline Palmar-plantar erythrodysesthesia syndrome & 14 & 12 & 0 & 2 & 0 \\
\hline Electrolyte derailment & 14 & 12 & 2 & 0 & 0 \\
\hline Vomiting & 7 & 7 & 0 & 0 & 0 \\
\hline QT-prolongation & 5 & 5 & 0 & 0 & 0 \\
\hline Mouth dryness & 5 & 5 & 0 & 0 & 0 \\
\hline Liver dysfunction & 2 & 0 & 2 & 0 & 0 \\
\hline
\end{tabular}

3.3. Lenvatinib Dosage. Dose reduction due to adverse effects resulted in a mean lenvatinib dose of $14 \pm 5 \mathrm{mg}$ per day (median: $14 \mathrm{mg}$; range: 4 to $24 \mathrm{mg}$ ). Four patients had no dose reduction at any time (9\%), while 39 patients (91\%) underwent (multiple) dose reductions in the course of disease. The maximum tolerated dose was $4 \mathrm{mg}$ in one patient (2\%), $10 \mathrm{mg}$ in 16 patients (37\%), $14 \mathrm{mg}$ in 18 patients (42\%), $18 \mathrm{mg}$ in 2 patients (5\%), $20 \mathrm{mg}$ in 1 patient (2\%), and $24 \mathrm{mg}$ in 5 patients (12\%), respectively. One patient (nr. 22) had a temporary dose reduction with a consecutive increase to $24 \mathrm{mg}$ lenvatinib daily again. Seven patients $(16 \%)$ discontinued lenvatinib treatment due to severe adverse events.

The maintenance dosage of lenvatinib had a significant effect ( $p=0.048$ ) on the overall survival of patients. Patients with a daily dose of $\leq 10 \mathrm{mg}$ lenvatinib $(n=17)$ had an overall survival of $63 \%$ (95\% CI: $39-86)$, while patients with a daily dose of $\geq 14$ mg lenvatinib $(n=26)$ reached an overall survival of $82 \%$ (95\% CI: 66-98) after 24 months, respectively (Figure 1).

3.4. TG Measurements. TG levels dropped by $75 \%$ after 1month treatment with lenvatinib and reached a minimum after 3 months with a decrease by $98 \%$. The change of mean TG levels before and after 1,3, and 12 months of therapy was statistically significant $(p<0.05)$, as were changes between the first and third month of lenvatinib therapy (Table 3, Figure 2).

No statistical difference in TG levels (baseline vs. 12 months) could be found in patients with a daily dose of $\leq 10 \mathrm{mg}$ lenvatinib and in patients with a daily dose of $\geq 14 \mathrm{mg}$, respectively.

\section{Discussion}

The clinical course of metastatic DTC shows clinically relevant variability with a range from patients with stable disease over many years to those with rapidly progressing disease and OS rates in the range of months upon diagnosis in least differentiated forms [10]. Real-world data are therefore of particular importance in RAI-refractory TC since they provide evidence on treatment efficacy and

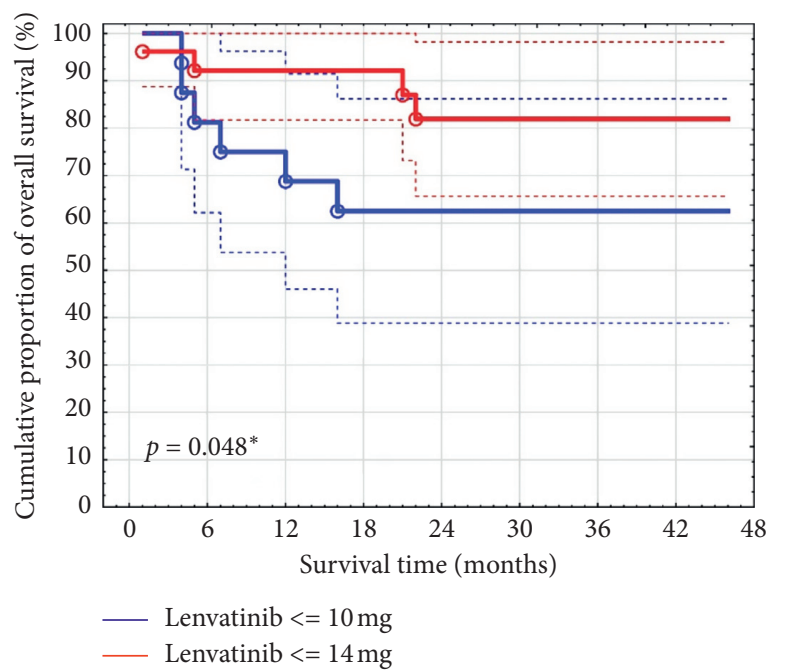

FIgURE 1: Overall survival with a daily dose of lenvatinib $\leq 10 \mathrm{mg}$ and $\geq 14 \mathrm{mg}$ (maintenance dose showed a statistically significant effect on survival).

TABLe 3: Thyroglobulin levels (mean $\pm S D$ ) at different time points.

\begin{tabular}{lcc}
\hline Thyroglobulin levels (ng/ml) & Mean & SD \\
\hline Max TG (before therapy) & $5,977.72$ & $13,692.36$ \\
$1 \mathrm{~m}$ & $1,515.15$ & $3,550.66$ \\
$3 \mathrm{~m}$ & 980.51 & $3,133.72$ \\
$12 \mathrm{~m}$ & $1,640.60$ & $3,566.92$ \\
$24 \mathrm{~m}$ & $2,643.75$ & $5,257.11$ \\
\hline
\end{tabular}

tolerability. The appropriate use of an anticancer drug in metastatic RAI-refractory TC in clinical routine should closely relate to the patient population tested in phase-III registration trials. This was true in our retrospective study in RAI-refractory TC patients exhibiting high rates of pulmonary ( $86 \%$ vs. $87 \%)$ and bone metastases ( $35 \%$ vs. $40 \%)$ when compared to the SELECT study $[18,23]$.

In the group of patients with progressive RAI-refractory DTC, lenvatinib significantly prolonged PFS to a median of 18.3 vs. 3.6 months in the placebo group [18]. In our analysis, the median PFS has not yet been reached. Seventy-one percent (95\% CI: 56-87) of our patients showed PFS after 24 


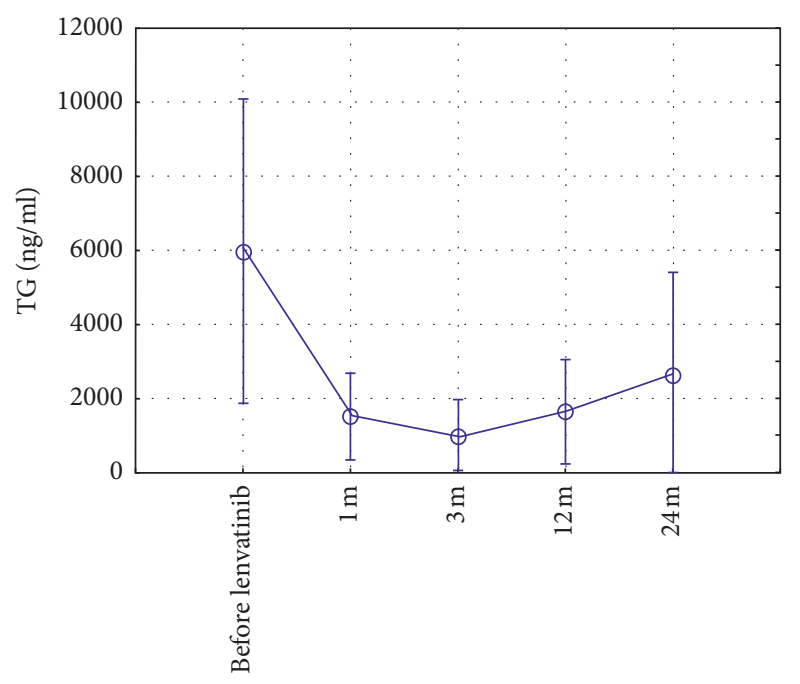

Ф. Mean with 95\% CI for mean

FIGURE 2: Change of thyroglobulin (TG) levels under therapy $(1 \mathrm{~m}=1$ month, $3 \mathrm{~m}=3$ months, $12 \mathrm{~m}=12$ months, and $24 \mathrm{~m}=24$ months).

months, and OS after 24 months amounted to 74\% (95\% CI: 60-88). Notably, our findings relay on a longer observation period than the registration study with a median duration of treatment with lenvatinib of 27.6 months as compared to 13.8 months in the registration trial [18].

In our study, the patient population was treated nearly four times longer than in other real-life studies in Europe and Japan [24-28] (Table 4).

OS was notably high in our patient collective, in spite of consisting of an elder population with extensive metastatic disease, including hepatic and brain metastases. Thereby, patients with brain metastases exhibited a worse outcome with a median OS of 12 months, while this was not seen in patients with liver metastases with a median OS of 32 months.

At the time of data cutoff, 10 patients (23\%) died due to progressive disease, and 7 patients (16\%) discontinued with lenvatinib, and the remaining 26 patients (61\%) were still on lenvatinib treatment.

Iodine deficiency was highly prevalent in the alpine region of Salzburg in Austria though its incidence was decreasing after the introduction of iodine supplementation [29]. Iodine deficiency has been associated with an increased proportion of FTC which might explain the predominance of FTC patients in our study (61\%) as compared to SELECT with $38.7 \%$. However, in agreement with the SELECT data, we found no impact of tumor histology on survival.

Every patient in our collective exhibited any form of adverse events of any grades, among which hypertension was most commonly seen $(71 \%, 8$ patients had grade 1 , and 11 patients each had grades 2 and 3, respectively). When considering grade 3 or 4 toxicities, only hypertension was common, but less frequently seen than in the SELECT study (26\% vs. $41.8 \%)$ [18].

Notably, a subanalysis of the latter demonstrated comparable efficacy in elderly subjects with a median age of 71 years in the presence of an increased toxicity profile [30].
The constellation of highly frequent adverse events in the presence of less high-grade toxicity might be associated with the finding of reduced maintenance dosages of lenvatinib employed throughout the observation period in our elder patient population. Decreased tolerability of any anticancer therapy might induce an earlier dose reduction in clinical routine than in clinical trials with their tight schedule of follow-up visits.

Weight loss $(70 \%)$ was also more common in our study population which might be due to the longer median observation period than in SELECT with a reported incidence of $46.4 \%$. Interestingly, weight loss was not always linked to decreased appetite, noted only in about $23 \%$ of our patients (vs. 50.2\% in the SELECT study). The reasons for weight loss are multiple and might relate to impaired intake due to dry mouth or painful food intake, clinically relevant proteinuria, and/or a catabolic state due to tumor cachexia.

Interestingly, very recently, a considerable proportion of RAI-refractory patients treated with lenvatinib has been shown to develop symptomatic biliary disorders [31]. These conditions might also contribute to significant weight loss. However, in our patient cohort, no patient presented with an onset of symptomatic biliary disease.

Persistent adverse events of any grade led to the discontinuation of treatment in 7 patients (16\% vs. $14.2 \%)$ in our collective. Any form of dose reduction was noted in $91 \%$ of our patients, which was more common than in the SELECT study with $67.8 \%$. The mean maintenance dose of lenvatinib was $14 \mathrm{mg}$ per day as compared to $17 \mathrm{mg}$ in the SELECT study. Dose reduction was mostly required during the first year of treatment, which is consistent with findings from both original and updated analysis of the SELECT patients $[23,32]$, in which the percentage of patients with grade $\geq 3$ lenvatinib-related adverse events increased by less than 5\% after 3 years of follow-up [23]. No new treatmentrelated deaths were reported in the updated analysis. SELECT and our data suggest that lenvatinib might be effective in patients with progressive RAI-refractory TC at lower than the standard dosage recommended for the use in RAI-refractory DTC.

Of note, lenvatinib has also been approved for the firstline treatment of advanced hepatocellular carcinoma (HCC) [33]. Interestingly, the maximum tolerable dose (MTD) for lenvatinib in HCC patients is significantly lower compared to RAI-refractory TC patients with a daily dose of $12 \mathrm{mg}$ /day for bodyweight $\geq 60 \mathrm{~kg}$ or $8 \mathrm{mg} /$ day for bodyweight $<60 \mathrm{~kg}$ [34]. The majority of HCC patients treated with lenvatinib needed dose reduction or drug withdrawal due to treatmentrelated adverse events [33]. Animal studies suggested that lenvatinib is predominantly metabolized in the liver and subsequently excreted via urine and feces [35]. Unmetabolized lenvatinib in urine and feces only accounts for $2.54 \%$ of the administered dose, hinting at a crucial role for liver metabolism in the elimination of lenvatinib [36]. In fact, HCC patients show a similar plasma concentration after multiple doses of $12 \mathrm{mg} /$ daily lenvatinib due to impaired liver function, like patients with solid tumors treated with multiple doses of $25 \mathrm{mg}$ /daily lenvatinib, resulting in similar areas under the curve (AUCs) [37]. In HCC patients, the 
Table 4: Real-life studies from Europe and Japan.

\begin{tabular}{|c|c|c|c|c|c|c|c|c|}
\hline Country & $N$ & $\begin{array}{c}\text { Age } \\
\text { (median; } \\
\text { years) }\end{array}$ & $\begin{array}{l}\text { Gender } \\
(\mathrm{m} / \mathrm{f})\end{array}$ & $\begin{array}{c}\text { Lenvatinib dose } \\
\text { median } \\
(\min -\max )\end{array}$ & $\begin{array}{c}\text { Median duration of } \\
\text { lenvatinib therapy } \\
\text { (months) }\end{array}$ & $\begin{array}{l}\text { PFS (median; } \\
\text { months) }\end{array}$ & $\begin{array}{l}\text { OS (median; } \\
\text { months) }\end{array}$ & $\begin{array}{l}\text { Observation period } \\
\text { (median; months) }\end{array}$ \\
\hline Italy [22] & 94 & 60 & $50 / 46$ & $\begin{array}{c}19.2 \mathrm{mg} \\
(10-24 \mathrm{mg})\end{array}$ & 5.9 & 10.8 & 23.8 & n.m. \\
\hline France [23] & 75 & 65 & $42 / 33$ & $\begin{array}{c}20 \mathrm{mg} \text { (n.m.- } \\
24 \mathrm{mg} \text { ) }\end{array}$ & 6 & 10 & Not reached & 7 \\
\hline Austria & 43 & 73 & $25 / 18$ & $14 \mathrm{mg}(4-24 \mathrm{mg})$ & 27.6 & Not reached & Not reached & 34.6 \\
\hline Japan [24] & 42 & 66 & $12 / 30$ & $\begin{array}{l}\text { Mean } 10 \mathrm{mg} \\
(4-24 \mathrm{mg})\end{array}$ & 14.9 & n.m. & n.m. & 15.4 \\
\hline $\begin{array}{l}\text { Netherlands } \\
{[25]}\end{array}$ & 39 & 72 & $20 / 19$ & $\begin{array}{c}18.6 \mathrm{mg} \\
(10-24 \mathrm{mg})\end{array}$ & 6.1 & 9.7 & 18.3 & n.m. \\
\hline $\begin{array}{l}\text { Switzerland } \\
{[26]}\end{array}$ & 13 & 72 & n. m. & n. $\mathrm{m} .(10-24 \mathrm{mg})$ & 9.98 & $\begin{array}{c}7.2 \\
\text { (estimated) }\end{array}$ & $\begin{array}{c}22.7 \\
\text { (estimated) }\end{array}$ & n.m. \\
\hline
\end{tabular}

n.m. = not mentioned.

AUC of lenvatinib is a reliable prognostic factor for dose reduction and occurrence of adverse events [34]. However, to the best of our knowledge, there have been no data on lenvatinib pharmacokinetic data in patients with TC, only data on solid tumors [38, 39].

An ongoing comparison phase-2 trial of lenvatinib (E7080) employing a starting dose of $18 \mathrm{mg}$ versus $24 \mathrm{mg}$ daily in RAI-refractory DTC patients addresses efficacy and safety profile in relation to dosage (211 study; NCT02702388).

Laboratory measurements of TG levels were used to assess disease progression biochemically in addition to standard imaging procedures. We detected a significant reduction in TG levels within a month by $75 \%$, which is in agreement not only with SELECT but also other trials with a range from 82 to $86 \%[40,41]$. A reduction could also be found in patients with low maintenance doses of lenvatinib ( $\leq 10 \mathrm{mg}$ daily). In an exploratory biomarker analysis of SELECT [42], the magnitude of change in TG levels appears to be associated with the objective response [42]. In our studies, a decrease in TG levels was also observed in patients with stable disease (SD) or progressive disease (PD), stressing the importance to validate other biomarkers [42] and/or imaging techniques for the assessment of treatment response.

Transient TG oscillations in the course of treatment were a frequent phenomenon that may not necessarily reflect morphologic tumor progression due to Werner et al. [41] and might be related to dose reductions or interruptions.

4.1. Limitations. A limitation of the study was the retrospective nature of our data collection. We included all histological subtypes of DTC in our clinical experience, as well as patients with ATC, who were given lenvatinib as an individual medical treatment due to tumor board decision.

Clinical, biochemical, and imaging analysis were performed at regular intervals though on behalf of the individual clinical judgement of the responsible physician. However, all centers applied similar protocols for the standard of care of DTC. In Austria, nuclear medicine departments are specialized for the management of thyroid cancer patients favoring similar diagnostic and therapeutic approaches which has also been published in a national consensus on the management of DTC by the use of TKI [6]. Notably, no patients were lost for follow-up indicating a high level of patient adherence.

It is another limitation of this study that either a PERCIST- or RECIST 1.1-based imaging analysis was used to evaluate tumor progression throughout different study centers. Moreover, PERCIST or RECIST 1.1. data were not available in a subset of study patients though the evaluation of the course of the disease in the respective center always comprised clinical, laboratory, and imaging-derived information.

Though FDG PET/CT has been shown to provide diagnostic and prognostic information in recurrent DTC due to its potential to identify RAI-refractory metastasis [43, 44], this technique is not uniquely recommended and employed in clinical routine.

Dose reductions were clinically required and performed based on the clinical judgement of the responsible specialist with differences regarding to their extent and duration and possible effects on the efficacy of the treatment.

\section{Conclusion}

Lenvatinib therapy showed sustained clinical efficacy in patients with metastatic RAI-refractory DTC in terms of year-long (progression-free) survival in clinical practice in Austria even when using lower reduced maintenance dosages. When employing lower dosages, toxicity is similar to the profile in controlled clinical trials, but mostly of lower grade, manageable, and clinically tolerable to the patient. The biomarker TG parallels the rapid clinical response during the first weeks of treatment. There is an obvious need for further controlled trials to assess the efficacy, tolerability, and toxicity profile of reduced daily dosage treatment regimen of lenvatinib in comparison to the standard dose of $24 \mathrm{mg}$ recommended in RAI-refractory DTC. 


\section{Data Availability}

The data used to support the findings of this study are available from the corresponding author upon request. For specific data from the research locations, contact G. Rendl (Salzburg, g.rendl@salk.at), A. Becherer (Feldkirch, Alexander.Becherer@lkhf.at), C. Trummer (Graz, christian.trummer@medunigraz.at), H. J. Gallowitsch (Klagenfurt, Hans-Juergen.Gallowitsch@kabeg.at), and M. Raderer (Wien, markus.raderer@meduniwien.ac.at).

\section{Disclosure}

This study was supported by an unrestricted research grant by Eisai.

\section{Conflicts of Interest}

The authors declare that they have no conflicts of interest.

\section{References}

[1] S. Austria and Ö. Krebsregister, Schilddrüse (C73)-krebsinzidenz (neuerkrankungen pro jahr), österreich ab 1983 (pdf), https:// www.statistik.at/web_de/statistiken/menschen_und_gesellschaft/ gesundheit/krebserkrankungen/schilddruese/index.html.

[2] S. Austria and Ö. Krebsregister, Schilddrüse (C73)krebsmortalität (sterbefälle pro jahr), österreich ab 1983 (pdf), https://www.statistik.at/web_de/statistiken/menschen_ und_gesellschaft/gesundheit/krebserkrankungen/schilddrues e/index.html.

[3] E. Grande, J. J. Diez, C. Zafon, and J. Capdevila, "Thyroid cancer: molecular aspects and new therapeutic strategies," Journal of Thyroid Research, vol. 2012, Article ID 847108, 10 pages, 2012.

[4] M. E. Cabanillas, D. G. McFadden, and C. Durante, "Thyroid cancer," The Lancet, vol. 388, no. 10061, pp. 2783-2795, 2016.

[5] G. Rendl, M. Rodrigues, G. Schweighofer-Zwink et al., "Clinicopathological characteristics of thyroid cancer in the federal state of Salzburg," Wiener Klinische Wochenschrift, vol. 129, no. 15-16, pp. 540-544, 2017.

[6] C. Lindner, J. Dierneder, G. Pall et al., "Treatment of patients with radioiodine refractory, differentiated thyroid carcinoma. A Consensus Statement]," Nuklearmedizin, vol. 54, pp. 125130, 2015.

[7] M. Luster, S. E. Clarke, M. Dietlein et al., "Guidelines for radioiodine therapy of differentiated thyroid cancer," European Journal of Nuclear Medicine and Molecular Imaging, vol. 35, no. 10, pp. 1941-1959, 2008.

[8] N. L. Busaidy and M. E. Cabanillas, "Differentiated thyroid cancer: management of patients with radioiodine nonresponsive disease," Journal of Thyroid Research, vol. 2012, Article ID 618985, 12 pages, 2012.

[9] M. Schlumberger, M. Brose, R. Elisei et al., "Definition and management of radioactive iodine-refractory differentiated thyroid cancer," The Lancet Diabetes \& Endocrinology, vol. 2, no. 5, pp. 356-358, 2014.

[10] C. Durante, N. Haddy, E. Baudin et al., "Long-term outcome of 444 patients with distant metastases from papillary and follicular thyroid carcinoma: benefits and limits of radioiodine therapy," The Journal of Clinical Endocrinology \& Metabolism, vol. 91, no. 8, pp. 2892-2899, 2006.
[11] B. R. Haugen, E. K. Alexander, K. C. Bible et al., "2015 American thyroid association management guidelines for adult patients with thyroid nodules and differentiated thyroid cancer: the American thyroid association guidelines task force on thyroid nodules and differentiated thyroid cancer," Thyroid, vol. 26, no. 1, pp. 1-133, 2016.

[12] F. Pacini, F. Basolo, R. Bellantone et al., "Italian consensus on diagnosis and treatment of differentiated thyroid cancer: joint statements of six Italian societies," Journal of Endocrinological Investigation, vol. 41, no. 7, pp. 849-876, 2018.

[13] M. S. Brose, C. M. Nutting, B. Jarzab et al., "Sorafenib in radioactive iodine-refractory, locally advanced or metastatic differentiated thyroid cancer: a randomised, double-blind, phase 3 trial," The Lancet, vol. 384, no. 9940, pp. 319-328, 2014.

[14] J. Matsui, Y. Yamamoto, Y. Funahashi et al., "E7080, a novel inhibitor that targets multiple kinases, has potent antitumor activities against stem cell factor producing human small cell lung cancer H146, based on angiogenesis inhibition," International Journal of Cancer, vol. 122, no. 3, pp. 664-671, 2008.

[15] O. Tohyama, J. Matsui, K. Kodama et al., "Antitumor activity of lenvatinib (e7080): an angiogenesis inhibitor that targets multiple receptor tyrosine kinases in preclinical human thyroid cancer models," Journal of Thyroid Research, vol. 2014, Article ID 638747, 13 pages, 2014.

[16] Eisai Europe Limited, Lenvima $4 \mathrm{Mg}$ Hard Capsules [Summary of Product Characteristics], Eisai Europe Limited, Hertfordshire, UK.

[17] Eisai Inc, Lenvima (Lenvatinib) Capsules, for Oral Use [Package Insert], Eisai Inc., Woodcliff Lake, NJ, USA, 2017.

[18] M. Schlumberger, M. Tahara, L. J. Wirth et al., "Lenvatinib versus placebo in radioiodine-refractory thyroid cancer," New England Journal of Medicine, vol. 372, no. 7, pp. 621-630, 2015.

[19] V. D. Tarasova and R. M. Tuttle, "A risk-adapted approach to follow-up in differentiated thyroid cancer," Rambam Maimonides Medical Journal, vol. 7, no. 1, 2016.

[20] E. A. Eisenhauer, P. Therasse, J. Bogaerts et al., "New response evaluation criteria in solid tumours: revised RECIST guideline (version 1.1)," European Journal of Cancer, vol. 45, no. 2, pp. 228-247, 2009.

[21] R. L. Wahl, H. Jacene, Y. Kasamon, and M. A. Lodge, "From RECIST to PERCIST: evolving considerations for PET response criteria in solid tumors," Journal of Nuclear Medicine, vol. 50, no. 1, pp. 122S-150S, 2009.

[22] U.S. Department of health and human services, National Institutes of Health, National Cancer Institute. Common Terminology Criteria for Adverse Events (CTCAE) https:// ctep.cancer.gov/protocoldevelopment/electronic_application s/docs/CTCAE_v5_Quick_Reference_8.5x11.pdf.

[23] A. G. Gianoukakis, C. E. Dutcus, N. Batty, M. Guo, and M. Baig, "Prolonged duration of response in lenvatinib responders with thyroid cancer," Endocrine-Related Cancer, vol. 25, no. 6, pp. 699-704, 2018.

[24] L. D. Locati, A. Piovesan, C. Durante et al., "Real-world efficacy and safety of lenvatinib: data from a compassionate use in the treatment of radioactive iodine-refractory differentiated thyroid cancer patients in Italy," European Journal of Cancer, vol. 118, pp. 35-40, 2019.

[25] A. Berdelou, I. Borget, Y. Godbert et al., "Lenvatinib for the treatment of radioiodine-refractory thyroid cancer in real-life practice," Thyroid, vol. 28, no. 1, 2018.

[26] C. Netwo, K. Sugino, N. Saito et al., "Efficacy and limitations of lenvatinib therapy for radioiodine-refractory differentiated 
thyroid cancer: real-world experiences," Thyroid, vol. 30, no. 3, pp. 214-221, 2020.

[27] M. D. Aydemirli, E. Kapiteijn, K. R. M. Ferrier et al., "Effectiveness and toxicity of lenvatinib in refractory thyroid cancer: Dutch real-life data," European Journal of Endocrinology, vol. 182, no. 2, pp. 131-138, 2020.

[28] C. Balmelli, N. Railic, M. Siano et al., "Lenvatinib in advanced radioiodine-refractory thyroid cancer - a retrospective analysis of the Swiss lenvatinib named patient program," Journal of Cancer, vol. 9, no. 2, pp. 250-255, 2018.

[29] A. Mostbeck, G. Galvan, P. Bauer et al., "The incidence of hyperthyroidism in Austria from 1987 to 1995 before and after an increase in salt iodization in 1990," European Journal of Nuclear Medicine and Molecular Imaging, vol. 25, no. 4, pp. 367-374, 1998.

[30] M. S. Zechmann, F. P. Worden, K. L. Newbold, M. Guo, and A. Hurria, "Effect of age on the efficacy and safety of lenvatinib in radioiodine-refractory differentiated thyroid cancer in the phase III SELECT trial," Journal of Clinical Oncology, vol. 35, no. 23, pp. 2692-2699, 2017.

[31] A. Nervo, A. Ragni, M. Gallo et al., "Symptomatic biliary disorders during lenvatinib treatment for thyroid cancer: an underestimated problem," Thyroid, vol. 30, no. 2, pp. 229-236, 2020.

[32] R. I. Haddad, M. Schlumberger, L. J. Wirth et al., "Incidence and timing of common adverse events in Lenvatinib-treated patients from the SELECT trial and their association with survival outcomes," Endocrine, vol. 56, no. 1, pp. 121-128, 2017.

[33] M. Kudo, R. S. Finn, S. Qin et al., "Lenvatinib versus sorafenib in first-line treatment of patients with unresectable hepatocellular carcinoma: a randomised phase 3 non-inferiority trial," The Lancet, vol. 391, no. 10126, pp. 1163-1173, 2018.

[34] T. Tamai, S. Hayato, S. Hojo et al., "Dose finding of lenvatinib in subjects with advanced hepatocellular carcinoma based on population pharmacokinetic and exposure-response analyses," The Journal of Clinical Pharmacology, vol. 57, no. 9, pp. 1138-1147, 2017.

[35] A.-C. Dubbelman, C. M. Nijenhuis, R. S. Jansen et al., "Metabolite profiling of the multiple tyrosine kinase inhibitor lenvatinib: a cross-species comparison," Investigational New Drugs, vol. 34, no. 3, pp. 300-318, 2016.

[36] A.-C. Dubbelman, H. Rosing, C. Nijenhuis et al., "Pharmacokinetics and excretion of 14C-lenvatinib in patients with advanced solid tumors or lymphomas," Investigational New Drugs, vol. 33, no. 1, pp. 233-240, 2015.

[37] M. Ikeda, T. Okusaka, S. Mitsunaga et al., "Safety and pharmacokinetics of lenvatinib in patients with advanced hepatocellular carcinoma," Clinical Cancer Research, vol. 22, no. 6, pp. 1385-1394, 2016.

[38] K. Yamada, N. Yamamoto, Y. Yamada et al., "Phase I doseescalation study and biomarker analysis of E7080 in patients with advanced solid tumors," Clinical Cancer Research, vol. 17, no. 8, pp. 2528-2537, 2011.

[39] S. Nakamichi, H. Nokihara, N. Yamamoto et al., "A phase 1 study of lenvatinib, multiple receptor tyrosine kinase inhibitor, in Japanese patients with advanced solid tumors," Cancer Chemotherapy and Pharmacology, vol. 76, no. 6, pp. 11531161, 2015.

[40] C. Masaki, K. Sugino, N. Saito et al., "Lenvatinib induces early tumor shrinkage in patients with advanced thyroid carcinoma," Endocrine Journal, vol. 64, no. 8, pp. 819-826, 2017.

[41] R. A. Werner, K. Lückerath, J. S. Schmid et al., "Thyroglobulin fluctuations in patients with iodine-refractory differentiated thyroid carcinoma on lenvatinib treatment - initial experience," Sci Rep, vol. 6, p. 28081, 2016.

[42] M. Tahara, M. Schlumberger, R. Elisei et al., "Exploratory analysis of biomarkers associated with clinical outcomes from the study of lenvatinib in differentiated cancer of the thyroid," European Journal of Cancer, vol. 75, pp. 213-221, 2017.

[43] M. J. Dong, Z. F. Liu, K. Zhao et al., "Value of 18F-FDG-PET/ PET-CT in differentiated thyroid carcinoma with radioiodinenegative whole-body scan: a meta-analysis," Nucl Med Commun, vol. 30, no. 8, pp. 639-650, 2013.

[44] R. J. Robbins, Q. Wan, R. K. Grewal et al., "Real-time prognosis for metastatic thyroid carcinoma based on 2-[18F] fluoro-2-deoxy-D-glucose-positron emission tomography scanning," The Journal of Clinical Endocrinology \& Metabolism, vol. 91, no. 2, pp. 498-505, 2006. 